\title{
Hydrodynamic Peculiarities of Fluid Flows in a Human Sigmoid Sinus by Data of Phase Contrast MR-angiography
}

\author{
A. Tulupov \\ Laboratory of translational brain research, The Institute \\ International Tomography Center of the Russian Academy \\ of Sciences \\ Novosibirsk State University \\ Russia
}

\begin{abstract}
A model of a human sigmoid sinus was studied using magnetic resonance imaging. MRI images were obtained in three transverse and two longitudinal sections at different levels. The findings indicate that, at the exit of the cranial cavity, this venous vascular structure forms a flow pattern with highly pronounced longitudinal vortices. Thereat, the flow rotation is formed by the complex asymmetric shape of the conduit.
\end{abstract}

Keywords-magnetic resonance imaging; flow quantification; sigmoid sinus

\section{INTRODUCTION}

Developing methods of medical diagnostics - ultrasound researches and MRI - enabled the study of fluid and gas flows in a human body. It is supposedly true that many medical institutions with necessary equipment perform such researches, especially on patients. However, the measurement on patients restricts somehow the experiment, for example it is impossible to increase the channel size, or to make a new one, long-term experiments are problematic. Thus, such researches need model experiments. In this case immediately appear the challenge of modeling of walls, especially elastic ones, and of model enter conditions. Most model investigations just repeat qualitatively the flows in living organisms. On the other hand, some physical simplified models are employed, for example channels with bifurcation [1], channels with local contractions simulating thrombosis [2].

\section{METHODS}

MRI study was made at $1.5 \mathrm{~T}$ system (Achieva, PHILIPS) with 2D and 3D phase contrast MR-angiography Quantitative Flow technique. Parameters of sequence were as follows: $\mathrm{TR}=14 \mathrm{~ms} ; \mathrm{TE}=8.3 \mathrm{~ms} ; \mathrm{FA}=15^{0}$; slice thickness $=3$ $\mathrm{mm}$. The paper presents the study of a flow in a sigmoid sinus - a section in a human vein located in the cranial cavity. The study was performed on a scale model with the similar Reynolds number $(\operatorname{Red} \approx 100)$.

\author{
V. Gorev \\ Khristianovich Institute of Theoretical and Applied \\ Mechanics SB RAS \\ Novosibirsk State University \\ Russia
}

\section{RESUlts}

Basing on the sigmoid sinus topographic anatomy of a healthy volunteer measured by the MRI, a computer model of a vessel channel was created. Then it was made from plastic by the rapid prototyping method. To form the needed boundary conditions at the model enter, a hydrodynamic tunnel with a set of channels of various lengths was manufactured. Using the straight channels of various lengths, one can have various flow profiles. If necessary, it is possible to manufacture a channel simulating the vessel section upstream from the studied section, to obtain the flow profile at the model enter which is maximally approaching to that one realizing in a human body.

In the present experiment, the Poiselle profile was used as the enter boundary conditions, because it is canonical and easily repeated, as well as the profile simulating the flow at the enter of this vessel in vivo. The sinus channel turns the blood flow approximately $90^{\circ}$ about its initial direction and directs it into the jugular vein. The channel shape is however redundantly complicated - instead of a simple return channel, the sigmoid sinus consists of two complex bends. As the measurements of velocity fields show, both bends imply the longitudinal swirl to the blood flow, all in one direction. Finally, at the sinus channel exit we observe an intensively rotating stream, the pitch of the spiral streamline in the flow core is approximately equal to the channel diameter, fig. 1. 


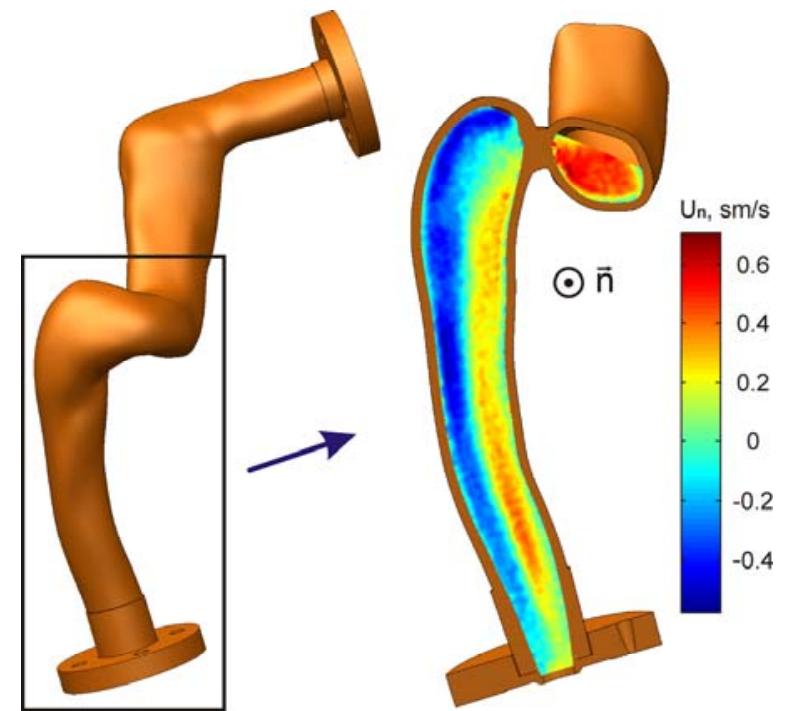

FIGURE I. THE MODEL OF A VESSEL CHANNEL AND NORMAL TO PLATE OF MEASUREMENTS VELOCITY FIELD DOWNSTREAM OF SECOND BEND.

\section{CONCLUSIONS}

The performed measurements revealed that the sigmoid sinus channel turns the flow blood direction by about $90^{\circ}$ relative to its original direction and directs it towards the jugular vein. The velocity field measurements indicate that, at its inlet from the cranial cavity, the sigmoid sinus forms a stream with highly pronounced longitudinal vorticity, all in the same direction at that. As a result, an intensity rotating stream is observed at the outlet of the sinus channel with the thread pitch of the helical current line in the stream core being approximately equal to the channel diameter. The qualitative and quantitative data collected in the course of the study on the characteristics of fluid flow in a model of human sigmoid sinus expand our fundamental understanding of the nature of the cerebral venous outflow, which will undoubtedly allow deeper exploration of the physiological mechanisms of cerebral hemodynamics and opens up a fundamentally new direction for further research in this area.

\section{ACKNOWLEDGMENTS}

This work was financially supported by the Russian Science Foundation (project no. 14-35-00020).

\section{REFERENCES}

[1] Leong, F.Y., Smith, K.A., Wang, C.-H., Secondary flow behavior in a double bifurcation. Physics of fluids, 21(4), Article number 043601, 2009.

[2] Petersson, S., Dyverfeldt, P., Gardhagen, R., Karlsson, M., Ebbers, T., Simulation of Phase-Contrast MRI of Turbulent Flow. Proc of the Workshop of International Society For Magnetic Resonance in Medicine, Sintra, Portugal, 11-13 Sept., 2009. 